\title{
INVARIANCE OF DOMAIN IN BANACH SPACES
}

\author{
NISHAN KRIKORIAN
}

\begin{abstract}
A continuous one-one map of the open unit disc of $l^{\infty}$ onto itself which is not a homeomorphism is constructed.
\end{abstract}

The Brouwer theorem on invariance of domain states that if $G$ is an open subset of Euclidean space $E$ and $f: G \rightarrow E$ is a continuous one-one map, then $f(G)$ is open and $f$ is a homeomorphism. This result has been extended to Banach spaces by Schauder [2] in the case when $f$ is of the form $I+\phi, \phi$ being completely continuous, and by Tromba [3] in the case when $f$ is a Fredholm map of index zero.

In general, invariance of domain fails in a Banach space since many Banach spaces are linearly homeomorphic to proper subspaces of themselves; furthermore Klee [1] has constructed a homeomorphism of separable Hilbert space onto a closed half-space. However, if the theorem is weakened so that $f(G)$ is already assumed to be open, does the result then hold in a Banach space? The answer is no as can be seen from the following extremely simple example.

Let $g_{n}: R \rightarrow R$ be the map with the graph

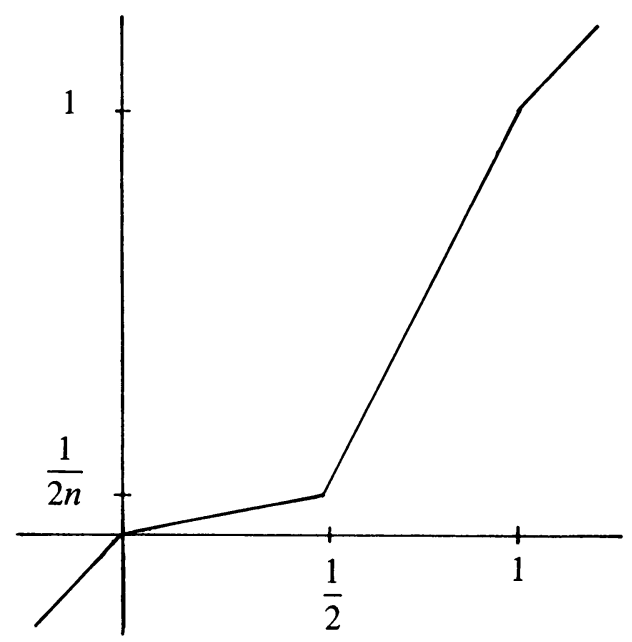

and let $G: l^{\infty} \rightarrow l^{\infty}$ be given by

Received by the editors May 1, 1975.

AMS (MOS) subject classifications (1970). Primary 47H99.

Copyright $\odot 1977$, American Mathematical Society 


$$
G\left(x_{1}, x_{2}, \ldots, x_{n}, \ldots\right)=\left(g_{1}\left(x_{1}\right), g_{2}\left(x_{2}\right), \ldots, g_{n}\left(x_{n}\right), \ldots\right) .
$$

Then $G$ is clearly one-one and takes the open unit disc onto itself. Furthermore $G$ is continuous, in fact Lipschitz continuous, since $\|G(x)-G(y)\|<$ $2\|x-y\|$. But $G^{-1}$ is not continuous at the origin since $(1 / n, 1 / n, \ldots) \rightarrow 0$ and $\left\|G^{-1}(1 / n, 1 / n, \ldots)\right\|>1 / 2$. Therefore $G$ is a continuous one-one map of the open unit disc of $l^{\infty}$ onto itself which is not a homeomorphism.

Also it is easy to see that $G$ can be made $C^{\infty}$ by properly rounding off corners. The derivative of $G$ will then have the form

$$
G^{\prime}\left(x_{1}, x_{2}, \ldots\right)\left(\varepsilon_{1}, \varepsilon_{2}, \ldots\right)=\left(g_{1}^{\prime}\left(x_{1}\right) \varepsilon_{1}, g_{2}^{\prime}\left(x_{2}\right) \varepsilon_{2}, \ldots\right)
$$

and will be a continuous transformation as long as $\left(g_{1}^{\prime}\left(x_{1}\right), g_{2}^{\prime}\left(x_{2}\right), \ldots\right) \in l^{\infty}$.

\section{REFERENCES}

1. V. L. Klee, Jr., Convex bodies and periodic homeomorphisms in Hilbert space, Trans. Amer. Soc. 74 (1953), 10-43. MR 14. 989.

2. J. Schauder, Invarianz des Gebietes in Funktionalräumen, Studia Math. 1 (1929), 123-139.

3. A. J. Tromba, Some theorems on Fredholm maps, Proc. Amer. Math. Soc. 34 (1972), 578-585. MR 45 \#7762.

Department of Mathematics, Northeastern University, Boston, MassachusetTs 02115 